\title{
Anesthesia for pericardial window in a pregnant patient with cardiac tamponade and mediastinal mass
}

\author{
[Anesthésie pour une fenêtre péricardique chez une femme enceinte qui présente
}

une tamponnade cardiaque et une masse médiastinale]

J. Anne Webster MD FRCPC, D. Dean Self MD FRCPC

Purpose: To present a case report of anesthesia for pericardial window surgery for acute cardiac tamponade in a patient with an anterior mediastinal mass in late pregnancy.

Clinical features: A 34-yr-old gravida 2, para I patient presented at 29 weeks gestation with dyspnea, orthopnea, chest pain, and cough. Investigations showed an anterior mediastinal mass due to Hodgkin's disease. A course of vinblastine at 31 weeks gestation resulted in symptomatic improvement but at 34 weeks gestation she developed an acute cardiac tamponade for which pericardial window drainage was required. Additional help and equipment were assembled in case of cardiopulmonary deterioration. Intraarterial pressure and continuous fetal monitoring were established and iv access was secured in both arms and the left foot. After awake fibreoptic intubation, spontaneous ventilation was maintained. Anesthesia consisted of local anesthetic infiltration of the anterior chest wall, supplemented with fentanyl, midazolam, and ketamine. The patient remained stable, was extubated fully awake, and then monitored in an intensive care area.

Conclusion: This patient presented with acute cardiac tamponade and an anterior mediastinal mass in late pregnancy, an unusual combination of challenges that requires a careful approach to anesthetic management.

Objectif : Décrire l'anesthésie pour une fenêtre péricardique pour tamponnade cardiaque aiguë chez une patiente en fin de grossesse qui présente une masse médiastinale antérieure.

Éléments cliniques : Une patiente de 34 ans, gravida 2, para I, a consulté à 29 sem de grossesse pour dyspnée, orthopnée, douleur thoracique et toux. Les examens ont montré une masse médiastinale antérieure liée à la maladie de Hodgkin. Le traitement par vinblastine, à 3 I sem, a diminué les symptômes, mais à 34 sem, une tamponnade cardiaque aiguë s'est développée, exigeant un drainage par fenêtre péricardique. Du personnel et du matériel supplémentaires ont été réunis en cas de détérioration cardio-pulmonaire. Le monitorage de la tension intra-artérielle et la surveillance fœetale continue ont été installés, ainsi qu'un accès iv aux deux bras et au pied gauche. Après l'intubation fibroscopique vigile, la ventilation spontanée a été maintenue. L'anesthésie comprenait l'infiltration d'un anesthésique local dans la paroi thoracique antérieure, complété par du fentanyl, du midazolam et de la kétamine. Les signes vitaux de la patiente sont demeurés stables; elle a été extubée complètement éveillée et gardée en observation à l'unité de soins intensifs.

Conclusion : Cette patiente présentait une tamponnade cardiaque aiguë et une masse médiastinale antérieure en fin de grossesse, une combinaison inhabituelle de défis qui exigent un traitement anesthésique prudent.

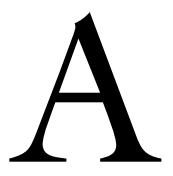

NESTHESIA for pericardial window surgery for relief of cardiac tamponade must take into account the impaired ventricular diastolic filling and associated decrease in stroke volume that result from increasing pericardial pressures. In this setting cardiac output is dependent upon maintenance of filling pressures, heart rate, and contractility. ${ }^{1-3}$ The presence of an anterior mediastinal mass adds the challenges of potential catastrophic obstruction of the airway, venous return, and pulmonary outflow. ${ }^{4-15}$ In addition, in late pregnancy there are the risks of pul-

From the Department of Anesthesia, Kelowna General Hospital, Kelowna, British Columbia, Canada.

Address correspondence to: Dr. J. Anne Webster, Department of Anesthesia, Victoria General Hospital, One Hospital Way, Victoria,

British Columbia V8Z 6R5, Canada. Phone: 250-727-4212; Fax: 250-721-9020; E-mail: jawebster@shaw.ca

No financial support has been received for this work.

Accepted for publication March 25, 2003.

Revision accepted June 11, 2003. 
monary aspiration of gastric contents, a potentially difficult airway, the compressive effect of a gravid uterus, and a second patient in the form of a fetus. ${ }^{6}$

\section{Case report}

A 34-yr-old $180 \mathrm{~cm} 93 \mathrm{~kg}$ gravida 2, para 1, diet controlled gestational diabetic patient developed progressive dyspnea, orthopnea, retrosternal pleuritic chest pain, and cough at 29 weeks gestation. Chest $x$-ray showed an anterior mediastinal mass. She was transferred for further investigation and treatment to a centre several hundred kilometres away because it was the nearest institution that could provide an appropriate level of care for a neonate of 29 weeks gestation, should it be required. A computed tomography (CT) scan of the thorax revealed a large mass, $14 \mathrm{~cm}$ in length and 6 to $7 \mathrm{~cm}$ in antero-posterior diameter, extending from the level of the thyroid to the superior margin of the right atrium and right ventricle, and causing moderate extrinsic compression of the left brachiocephalic vein, but causing no obvious airway compression. A flow volume loop was normal. An anterior mediastinotomy for tissue diagnosis was performed at the referral centre. The anesthetic given there consisted of inhalation induction of general anesthesia with sevoflurane and cricoid pressure followed by endotracheal intubation and maintenance of spontaneous ventilation. Upon completion of staging procedures, a diagnosis of stage IIA nodular sclerosing Hodgkin's disease was made. The patient was given two $i m$ doses of betamethasone to enhance fetal lung maturity. A trial of single agent vinblastine chemotherapy, with the first course given at 31 weeks, resulted in considerable symptomatic improvement, but a followup chest $x$-ray at 33 weeks gestation showed no change in the size of the mass. She was instructed to appear one week later for another chest $x$-ray but instead, because she felt so well, returned from the referral centre back to her home city against advice. A few days after reaching 34 weeks gestation she suddenly developed increasing dyspnea and orthopnea. On examination in the sitting position, the heart rate was 110 beats. $\mathrm{min}^{-1}$, the blood pressure was $110 / 80$ $\mathrm{mmHg}$ with a pulsus paradoxus of 15 to $20 \mathrm{mmHg}$, the jugular veins were distended, the trachea was midline, and breath sounds were normal. An echocardiogram showed a pericardial effusion and right atrial collapse, confirming cardiac tamponade. Pericardial window drainage was planned after a discussion between the thoracic surgeon and radiologist determined that because of the patient's size and orthopnea, and the relatively small size of effusion, the risks of percutaneous techniques exceeded those of open drainage. Transport to a centre with cardiopulmonary bypass was ruled out because of the potential for sudden catastrophic cardiopulmonary deterioration in transit to the nearest facility, which was $380 \mathrm{~km}$ distant over high mountains in winter conditions.

A second anesthesiologist, obstetrician, and pediatrician were recruited and equipment was assembled for rigid bronchoscopy and Cesarean delivery if necessary. The patient was given $30 \mathrm{~mL}$ of sodium citrate and brought to the operating room in the sitting position receiving oxygen by mask. Pulse oximetry, electrocardiography, and continuous fetal monitoring were initiated. In addition to an existing arm $i v$ access site, large bore $i v$ catheters were established in the opposite arm and left foot. An arterial pressure monitoring catheter was placed in the right radial artery under local anesthesia. Incremental doses of midazolam and fentanyl were given while topical anesthesia was applied to the airway. Airway anesthesia was accomplished with gargled, then expelled, topical $4 \%$ lidocaine, followed by $2 \%$ lidocaine spray applied to the pharynx and $3 \mathrm{~mL}$ $2 \%$ lidocaine administered subglottically through the fibreoptic bronchoscope. Awake fibreoptic intubation was followed by bronchoscopy, which revealed no obvious airway compression. End-tidal gas monitoring was instituted. Spontaneous ventilation was maintained with gentle assistance as necessary to control hypercapnia as seen on end-tidal gas measurements. Small doses of $i v$ ketamine were titrated to achieve amnesia and analgesia in an anxious patient. The patient was placed gradually in a recumbent position with her head slightly elevated and her right side tilted down. The incision site was infiltrated with approximately $7 \mathrm{~mL}$ of $2 \%$ carbonated lidocaine. Sevoflurane (up to $1 \%$ ) was used in response to movement and increased blood pressure to reduce the possibility of patient awareness. During the procedure $3 \mathrm{~L}$ of $i v$ crystalloid solution were infused. When the surgeon opened the pericardium to drain $425 \mathrm{~mL}$ of sanguineous fluid, the systolic arterial pressure increased 10 to $15 \mathrm{mmHg}$. A total of $110 \mathrm{mg}$ of ketamine, $3 \mathrm{mg}$ of midazolam and $150 \mu \mathrm{g}$ of fentanyl had been given by the completion of the surgery. No significant airway obstruction, hemodynamic decompensation, or fetal distress occurred. The patient was extubated when fully awake and was monitored in an intensive care area. Four days later labour was induced. Effective epidural analgesia was established early in labour to allow for rapid provision of regional anesthesia in the event of emergency Cesarean section. The patient proceeded to an uneventful vaginal delivery of a healthy female infant and resumed treatment for Hodgkin's disease the following day. 


\section{Discussion}

Cardiac tamponade and anterior mediastinal mass can present with very similar clinical features, including cough, dyspnea, orthopnea, jugular venous distention, and pulsus paradoxus. ${ }^{5}$ In this patient the rapid onset of symptoms coinciding with the development of cardiac tamponade against a background of stable mediastinal mass suggested tamponade as the primary cause of her deterioration.

In cardiac tamponade, an increase in endogenous catecholamines causes increases in heart rate, contractility, and systemic vascular resistance. This compensates for impaired ventricular diastolic filling and the resultant decreases in stroke volume, blood pressure, and cardiac output. ${ }^{1-3}$ Right atrial or right ventricular collapse in the presence of a pericardial effusion on echocardiography confirms the diagnosis of tamponade. ${ }^{1}$ In the presence of tamponade, failure to maintain heart rate, contractility, and ventricular filling pressures may result in dramatic decreases in cardiac output, causing cardiovascular collapse. ${ }^{1}$ Since most general anesthetic agents cause myocardial depression and systemic vasodilation, and positive pressure ventilation decreases venous return, local anesthesia is recommended for relief of tamponade that is severe enough to cause hypotension. ${ }^{1,16,17}$ In less severe cases, several authors suggest the use of ketamine for analgesia or anesthesia because ketamine causes less respiratory depression and tends to support heart rate, contractility, and systemic vascular tone compared to other drugs. ${ }^{1,2,18}$ Spontaneous ventilation and intravascular volume expansion may help maintain and enhance venous return and hence help maintain and enhance ventricular filling pressures. ${ }^{1-3}$

An anterior mediastinal mass may cause sudden catastrophic obstruction of major airways or major vessels if general anesthesia is induced. ${ }^{4-15}$ These complications can occur even in patients who are asymptomatic while awake. ${ }^{6,7,12-14}$ Airway obstruction, which can occur at any stage of anesthesia, ${ }^{7,9,12,13,15}$ is the most common problem encountered. ${ }^{9}$ The causes of airway obstruction are several: the supine position allows the weight of the mass to lie directly over the major airways; ${ }^{13,15}$ anesthesia causes a decrease in chest wall ${ }^{13}$ and bronchial muscle tone thereby decreasing the tethering effect on airway diameter; ${ }^{4,12,15}$ loss of spontaneous ventilation decreases the airway dilating effect of negative intrathoracic pressure; ${ }^{4,12,15}$ and positive pressure ventilation paradoxically may worsen the obstruction by causing increased post-obstructive turbulence. ${ }^{15}$ Attempts to identify patients at risk of perioperative airway obstruction have used CT measurements of airway cross-sectional areas ${ }^{4,7,14}$ and flow volume loops. ${ }^{12,14}$ In children,
Azizkhan et al. found a correlation between severity of symptoms and presence and degree of airway compression on CT scan. Based on his series of 50 children he suggested that a $35 \%$ or greater decrease in airway cross-sectional area indicated significant risk and that general anesthesia should be avoided in these patients. ${ }^{4}$ A flow volume loop, however, may reveal a decreased peak expiratory flow rate and a plateau shape to the expiratory limb of the loop, indicating significant intrathoracic airway obstruction, ${ }^{13}$ despite lack of CT evidence of airway narrowing. ${ }^{12,14}$ Neuman points this out and emphasizes the value of the flow volume loop as a dynamic measure of a dynamic process as opposed to a static measure as provided by CT scanning. ${ }^{12}$

By mechanisms similar to those for airway obstruction, general anesthesia in the presence of an anterior mediastinal mass can precipitate cardiac, superior vena cava, and pulmonary artery compression, although these problems are less common and less well characterized in the literature. Compression of major vessels can cause hypoxemia, hypotension, and cardiovascular collapse. ${ }^{6,9,13}$ Mass effects on major vessels may be evaluated by supine and upright echocardiography. ${ }^{5,6,8,12}$

Management of anesthesia in the presence of an anterior mediastinal mass is directed toward avoiding critical airway or major vessel obstruction. Lower limb $i v$ access allows effective fluid and drug administration in the event of superior vena cava obstruction ${ }^{6,7,9,13}$ and continuous invasive arterial pressure monitoring facilitates prompt detection of cardiovascular compromise. Where possible, local, or regional anesthesia, provided the effect of vasodilation on venous return is considered, should be used. . $7,8,10,12-14,19$ Ketamine is useful for sedation or anesthesia because it causes less respiratory depression and maintains chest wall and peripheral vascular tone better than most other agents. ${ }^{9,18,19}$ Spontaneous ventilation should be maintained as much as possible. ${ }^{4,5,12-15,19}$ If the site of airway compression identified on CT scan is relatively localized, it may be amenable to bypass with an endotracheal tube or rigid bronchoscope. ${ }^{4,7,9}$ Head up, lateral, or prone positioning can help shift the weight of the mass away from vital structures. ${ }^{4-6,9,13}$ In patients with significant CT, flow volume loop, or echocardiographic abnormalities, in whom general anesthesia is required, preoperative preparation for immediate cardiopulmonary bypass should be considered. ${ }^{4-7,9,12,13,20}$ If time permits, preoperative chemotherapy or radiotherapy may be used to shrink malignant anterior mediastinal masses to make anesthesia safer. ${ }^{9,13,15}$ However, this may seriously impair the ability to make an accurate tissue diagnosis if one has not been obtained. ${ }^{12,14,15}$ 
Since the patient was pregnant we intubated her awake to secure and protect her airway prior to obtunding the central nervous system with ketamine ${ }^{18}$ and sevoflurane. The positioning we used not only facilitated left anterior mini-thoracotomy, but shifted the uterus away from the inferior vena cava, allowing us to avoid compounding impaired venous return due to possible compression of the superior vena cava. In the event of cardiopulmonary deterioration or fetal distress, we were prepared for immediate Cesarean delivery to facilitate resuscitation of mother and infant.

In summary this patient presented with a symptomatic anterior mediastinal mass in late pregnancy and then developed acute cardiac tamponade requiring surgical drainage. Our approach of direct arterial pressure and fetal monitoring, use of lower limb iv access, awake intubation, partial head up and right lateral positioning, use of ketamine and local anesthesia, and maintenance of spontaneous ventilation was designed to minimize the potential adverse pathophysiologic consequences of anesthesia in the context of these three problems.

\section{References}

1 Ammar T, Reich DL, Kaplan JA. Uncommon cardiac diseases. In: Benumof JL (Ed.). Anesthesia and Uncommon Diseases, 4th ed. Philadelphia: WB Saunders Co.; 1998: 70-122.

2 Lake CL. Anesthesia and pericardial disease. Anesth Analg 1983; 62: 431-43.

3 Stoelting RK, Dierdorf SF. Pericardial diseases. In: Stoelting RK, Dierdorf SF (Eds.). Anesthesia and CoExisting Disease, 4th ed. New York: Churchill Livingston Inc.; 2002: 135-42.

4 Azizkhan RG, Dudgeon DL, Buck JR, et al. Life-threatening airway obstruction as a complication to the management of mediastinal masses in children. J Pediatr Surg 1985; 20: 816-22.

5 Dilworth KE, McHugh K, Stacey S, Howard RF. Mediastinal mass obscured by a large pericardial effusion in a child: a potential cause of serious anaesthetic morbidity. Paediatr Anaesth 2001; 11: 479-82.

6 Ferrari LR, Bedford RF. Anterior mediastinal mass in a pregnant patient: anesthetic management and considerations. J Clin Anesth 1989; 1: 460-3.

7 Goh MH, Liu XY, Goh YS. Anterior mediastinal masses: an anaesthetic challenge. Anaesthesia 1999; 54: 670-4.

8 Greengrass $R$. Anaesthesia and mediastinal masses (Letter). Can J Anaesth 1990; 37: 596.

9 Mackie AM, Watson CB. Anaesthesia and mediastinal masses. A case report and of the literature. Anaesthesia 1984; 39: 899-903.

10 Martin WJ. Cesarian section in a pregnant patient with an anterior mediastinal mass and failed supradiaphragmatic irradiation. J Clin Anesth 1995; 7: 312-5.

11 McMahon CC, Rainey L, Fulton B, Conacher ID. Central airway compression. Anaesthetic and intensive care consequences. Anaesthesia 1997; 52: 158-62.

12 Neuman GG, Weingarten AE, Abramowitz RM, Kushins LG, Abramson AL, Ladner W. The anesthetic management of the patient with an anterior mediastinal mass. Anesthesiology 1984; 60: 144-7.

13 Pullerits J, Holzman R. Anaesthesia for patients with mediastinal masses. Can J Anaesth 1989; 36: 681-8.

14 Shamberger RC. Preanesthetic evaluation of children with anterior mediastinal masses. Semin Pediatr Surg 1999; 8: 61-8.

15 Sibert KS, Biondi JW, Hirsch NP. Spontaneous respiration during thoracotomy in a patient with a mediastinal mass. Anesth Analg 1987; 66: 904-7.

16 Breen PH, MacVay MA. Pericardial tamponade: a case for awake endotracheal intubation (Letter). Anesth Analg 1996; 83: 658.

17 Stanley TH, Weidauer HE. Anesthesia for the patient with cardiac tamponade. Anesth Analg 1973; 52: 110-4.

18 Stoelting $R K$. Nonbarbiturate induction drugs. In: Stoelting R (Ed.). Pharmacology and Physiology in Anesthetic Practice, 3rd ed. Philadelphia, Pennsylvania: Lippincott-Raven; 1999: 104-57.

19 Tinker TD, Crane DL. Safety of anesthesia for patients with anterior mediastinal masses: 1 (Letter). Anesthesiology 1990; 73: 1060.

20 Chan YK, Ng KP, Chiu CL, Rajan G, Tan KC, Lim $\Upsilon C$. Anesthetic management of a parturient with superior vena cava obstruction for cesarean section. Anesthesiology 2001; 94: 167-9. 